\title{
Complex regional pain syndrome
}

\author{
The authors explore how doctors in primary care can identify, approach, and refer patients with \\ regional pain syndrome
}

\author{
Fernanda B Fukushima assistant professor ${ }^{1}$, Dailson M Bezerra PhD student ${ }^{1}$, Paulo J F Villas \\ Boas associate professor ${ }^{2}$, Adriana $\mathrm{P}$ Valle associate professor ${ }^{2}$, Edison I O Vidal assistant \\ professor $^{2}$
} 'Anesthesiology Department, Universidade Estadual Paulista (UNESP), 18618-970, Botucatu, SP, Brazil; ${ }^{2}$ Internal Medicine Department, Universidade
Estadual Paulista (UNESP), 18618-970, Botucatu, SP, Brazil

Complex regional pain syndrome (CRPS) is characterised by constant regional neuropathic pain that is usually associated with abnormal sensory, autonomic, motor and/or trophic changes. ${ }^{1}$ Though it usually develops after trauma to a limb, in CRPS pain is disproportionate in time or intensity to the usual course of pain after injury. There are two subtypes of CRPS: in type I no overt nerve lesion can be identified; in type II definite nerve injury is evident.

CRPS has had various names including causalgia, reflex sympathetic dystrophy, Sudeck's atrophy, algoneurodystrophy, and shoulder-hand syndrome. All those terms were replaced by CRPS after a 1993 conference by the International Association for the Study of Pain (IASP), which aimed at clarifying and providing more uniformity for diagnosis. Only recently has it begun to feature in more general medical textbooks, and most clinicians have not been trained to recognise it. ${ }^{2}$

The pathophysiology of CRPS is multifaceted and remains incompletely understood. Several lines of evidence point towards disturbances involving abnormal response to tissue injury, peripheral and central pain sensitisation processes, neurogenic inflammation, endothelial dysfunction, disturbed sympathetic-afferent coupling, hyperalgesic priming, somatosensory cortical reorganisation, genetic predisposition, and even some degree of autoimmunity. ${ }^{3}$

\section{Methods}

We searched Medline, the Cochrane Library, and the website of the International Association for the Study of Pain (www. iasp-pain.org) using the terms "complex regional pain syndrome", "causalgia", "reflex sympathetic dystrophy", "Sudeck's atrophy", "algodystrophy", "algoneurodystrophy", and "reflex neurovascular dystrophy". We searched the reference lists of selected articles for further relevant references. Our search was aimed at articles on the epidemiology, pathophysiology, natural course, diagnosis, and treatment of CRPS. We considered original and review articles, with special emphasis on systematic reviews and clinical guidelines. We also drew from our own personal libraries.

\section{How common is complex regional pain syndrome?}

The incidence of CRPS is uncertain. To date there have been only two retrospective population based studies. One study from the United States in 2003 found an incidence of 5.46 cases per 100000 person years and a prevalence of 20.57 cases per 100 000 people in $1999 .{ }^{4}$ A 2007 study from the Netherlands observed an incidence of 26.2 cases per 100000 person years. ${ }^{5}$ In both studies the female to male ratio ranged from 3.4-4.0:1, and the peak incidence was seen in those aged 50-70. As both studies were based on retrospective review of database or medical record codes, however, the incidence figures might be underestimates.

Two recent well designed studies that prospectively assessed the incidence of CRPS after a fracture found that it occurred in $3.8 \%$ of 1549 patients within four months after a wrist fracture ${ }^{6}$ and in $7 \%$ of 596 patients within one year after wrist, scaphoid, ankle, or metatarsal fracture. ${ }^{7}$

\section{What are the clinical features?}

CRPS usually develops after a fracture, soft tissue injury, or surgical trauma; it can, however, be triggered by prolonged immobilisation, disorders of central nervous system (such as stroke), and visceral lesions (such as myocardial infarction). In about $10 \%$ of cases no apparent precipitating event can be identified. $^{235}$ 


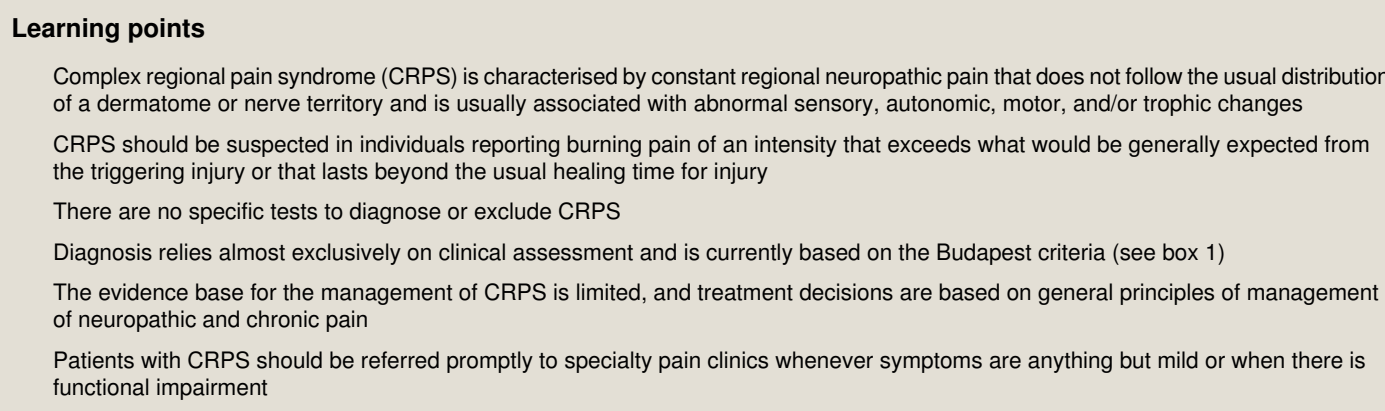

Part of the diagnostic challenge of CRPS derives from its diverse presentations, varying between patients and within individuals over time. Its key clinical features are spontaneous pain, vasomotor changes, motor abnormalities, hyperalgesia (that is, exaggerated pain to a painful stimulus such as pinprick), and/or allodynia (that is, pain elicited by a normally non-painful stimulus such as light touch). Those features persist beyond the expected time for recovery from the original injury, usually have a distal preponderance, and do not follow the usual distribution of a dermatome or nerve territory. More bizarre presentations include spreading of pain and other disturbances to other limbs and the occurrence of skin ulceration. ${ }^{8}$ It is important to emphasise that the intensity of signs and symptoms can fluctuate over short periods of time and that in the first weeks of CRPS an individual patient might present with a particular set of signs and symptoms (such as pain, warmth, redness, and swelling), which can later evolve gradually into another clinical picture (such as pain, cold, cyanosis, and atrophy). The concept of three sequential stages in the evolution of CRPS, however, is no longer considered valid. ${ }^{9}$

The typical pattern of CRPS pain is neuropathic, being described by patients as burning, tingling, stabbing, numbness, or like an electric shock. This is in contrast with nociceptive pain patterns, which are usually described as sharp, aching, and/or throbbing pain. Yet clinicians must be aware that both neuropathic and nociceptive pain patterns often coexist in the same region affected by CRPS as a myofascial component has been reported. ${ }^{10}$

Vasomotor and sudomotor changes manifest as changes in temperature, skin colour, moistness, or oedema. ${ }^{2}$ These changes are diverse and can evolve with time. The affected region might be warm and pink or cold, brownish, mottled, and/or cyanotic (figure $\Downarrow$ ). The skin can become atrophic and shiny. There can be asymmetry or changes (increases or decreases) in sweating and in hair and nail growth compared with the unaffected side. Nails can grow to be hypertrophic or atrophic, brittle, and ridged (figure $\downarrow$ ). Abnormal motor function can manifest as motor weakness, difficulty in initiating movements, tremor, muscle spasms, and even dystonia. Finally, patients can experience disturbances in body perception including neglect and distorted mental image of the affected body part.

Unfortunately, given such a diverse presentation and the lack of a simple neuroanatomical explanation for these signs and symptoms, many professionals come to believe that patients might be malingering or somatising. On the contrary, there is evidence that neither psychological profile nor previously existing depression or anxiety predisposes individuals to CRPS. ${ }^{3}$

\section{What is the natural course of CRPS?}

Current knowledge about the natural course of CRPS is limited. In a retrospective cohort of 102 patients with CRPS, identified from a Dutch general practitioners' database, and evaluated directly by interview and examination, the mean time from the triggering injury was 5.8 years, $30 \%$ considered themselves as completely recovered, $16 \%$ still reported severe progressive disease, and 54\% were stable. Among the 54 patients who were working before they developed CRPS, $41 \%$ had resumed their former job entirely and $28 \%$ returned to work with some adjustment, whereas $31 \%$ were completely incapable to work. ${ }^{11}$ In that study the following characteristics were significantly associated with worst outcome: having the upper extremity affected, having a triggering event other than a fracture, and displaying "cold CRPS." CRPS has usually been classified as either cold or warm according to the predominating skin temperature of the affected limb(s) at disease onset. De Mos and colleagues, ${ }^{11}$ however, defined cold CRPS as CRPS with self reported predominantly cold skin in the affected limb when the patient was assessed for the study, which happened at least two years after disease onset. Rates of recovery might be higher as the study might not have identified many cases of mild CPRS with early resolution. For instance, in another retrospective population based study in 55 of 74 cases of CRPS the patient experienced complete pain resolution. ${ }^{4}$

\section{How is it diagnosed?}

Currently the so called "Budapest criteria" (box 1) are recommended for the diagnosis of CRPS. ${ }^{89}$ In an international multisite validation study analysing the accuracy of those criteria to distinguish between CRPS type I and other neuropathic pain syndromes within the context of specialty clinics, the clinical criteria were shown to be $99 \%$ sensitive and $68 \%$ specific, ${ }^{12}$ whereas the research criteria were found to be $78 \%$ sensitive and $79 \%$ specific.

Although there are no specific diagnostic tests for CRPS, several ancillary tests are useful to rule out other diagnoses. ${ }^{2}$ Tests for full blood count, $\mathrm{C}$ reactive protein, erythrocyte sedimentation rate, and serum autoantibodies are helpful to exclude infection and rheumatologic disorders (for instance, when swollen and warm extremities could denote cellulitis or arthritis, or when vasospasm of the fingers could indicate Raynaud's phenomenon secondary to autoimmune disorders), as those results are expected to be normal in those with CRPS. Duplex scanning is valuable to exclude deep vein thrombosis or peripheral arterial obstruction in selected patients (such as those with an acute episode of asymmetrical limb oedema or patients with resting leg pain associated with decreased or absent peripheral pulses and risk factors for atherosclerotic disease). Standard radiographs might show patchy demineralisation that is most marked in periarticular areas; nonetheless those findings are neither sensitive nor specific. Results of electroneuromyography (nerve conduction studies) and electromyography are usually normal in type I CRPS but can be abnormal in type II. Electroneuromyography, however, should not be ordered 
Box 1: Budapest clinical ${ }^{*}$ diagnostic criteria for complex regional pain syndrome (adapted from Harden et al $^{12}$ )

Each of the following criteria must be met:

1. Patients must report continuing pain that is disproportionate in time or degree to the usual course of pain after any trauma or other inciting event $†$

2. Patients must report at least one symptom in three of the four following categories:

(a) Sensory: hyperalgesia (that is, exaggerated pain to a painful stimulus, such as pinprick) and/or allodynia (that is, pain elicited by a normally non-painful stimulus, such as light touch)

(b) Vasomotor: skin colour and/or temperature changes/asymmetry

(c) Sudomotor/oedema: swelling and/or sweating changes or asymmetry

(d) Motor/trophic: weakness, tremor, dystonia, decreased range of motion and/or trophic changes/asymmetry involving nails, skin and/or hair

3. Patients must display at least one sign at the time of assessment in two or more of the same four categories listed above

4. Signs and symptoms must not be better explained by another diagnosisł

*There are two versions of the Budapest criteria: the "clinical criteria" (described above and aimed at maximising sensitivity) and the "research criteria" (aimed at more equally balancing sensitivity and specificity). The "research criteria" differ from the "clinical criteria" described only by the requirement that at least one symptom in each of those four categories be present instead of at least one symptom in three of the four categories.

†As noted in the text, in about $10 \%$ of cases no apparent precipitating injury can be identified, though in fact a very mild inciting event might have occurred. ${ }^{235}$

$\ddagger$ According to those criteria there are three possible diagnostic categories for CRPS. The first two categories are CRPS types II and I according to the presence or absence of peripheral nerve injury, respectively. A third category, CRPS-not otherwise specified (CRPS-NOS) denotes those patients who do not fulfil all of the clinical or research criteria for CRPS but whose signs and symptoms cannot be better explained by other conditions.

routinely because testing might exacerbate the pain of CRPS and distinguishing between the subtypes rarely alters management. If it is suspected that type II CRPS has arisen as a result of a remediable nerve injury (such as nerve compression by a neuroma or perioperative scar tissue), then electroneuromyography and surgical referral might be appropriate.

\section{How is it managed?}

A recently updated Cochrane review of systematic reviews on interventions for the treatment of CRPS concluded that the evidence base for most treatments for CRPS is "of low to very low quality and cannot be regarded as reliable."13

So, what can be done to help patients? Firstly, while acknowledging that currently there is little certainty about what works for patients with CRPS, clinicians must carefully examine with their patients the risk and cost-benefit ratios of different treatment options, taking into account the patient's preferences and individual characteristics. Secondly, clinicians can draw from higher quality evidence available for the treatment of other forms of neuropathic pain and from lesser quality but more specific interventions aimed at CRPS, while recognising that every approach to treatment should be considered tentative. Thirdly, all but those patients with mild forms of CRPS should be referred for assessment at a specialty pain clinic. Such clinics can usually offer integrated interdisciplinary pain care as well as some invasive procedures for selected patients with severe refractory pain. $^{12}$

Tricyclic antidepressants, serotonin-norepinephrine reuptake inhibitors, the anticonvulsants gabapentin and pregabalin, and topical lidocaine are considered preferred treatments for the general management of neuropathic pain that can be tried with CRPS patients following available guidelines. ${ }^{14} 15$

Bisphosphonates have been endorsed by some clinical guidelines based on some evidence of benefit in five small clinical trials. ${ }^{89}$ Several questions, however, remain over the use of bisphosphonates in patients with CRPS, such as which characteristics of patients are predictive of response and which doses, frequencies, and durations of treatment are optimal.

Finally, some general principles that are considered effective for the management of other chronic pain conditions in general are recommended..$^{8}$ They include provision of education for patients and their families about CRPS and chronic pain management principles such as pacing, goal setting, and relaxation (box 2). Multidisciplinary pain management programmes focusing on functional restoration also seem a logical step in the management of such a complex, heterogeneous, and often debilitating disease.

Contributors: FBF and EIOV conceived the article and drafted the first version of the manuscript. PJFVB and APV provided the general practice perspective about CRPS. DMB provided additional specialist perspective in pain medicine. All authors revised the manuscript critically for important intellectual content and approved the final version. FBF is guarantor.

Competing interests: We have read and understood the BMJ policy on declaration of interests and declare the following interests: none.

Provenance and peer review: Not commissioned; externally peer reviewed.

Patient consent: Informed consent was given by the patient in the figure.

1 International Association for the Study of Pain. Classification of chronic pain. 2nd ed. www.iasp-pain.org/AM/Template.cfm?Section=Publications\&Template=/CM/HTMLDisplay cfm\&ContentID=2687.

2 Albazaz R, Wong YT, Homer-Vanniasinkam S. Complex regional pain syndrome: a review. Ann Vasc Surg 2008;22:297-306.

3 Marinus J, Moseley GL, Birklein F, Baron R, Maihöfner C, Kingery WS, et al. Clinical features and pathophysiology of complex regional pain syndrome. Lancet Neurol 2011;10:637-48.

4 Sandroni P, Benrud-Larson LM, McClelland RL, Low P. Complex regional pain syndrome type I: incidence and prevalence in Olmsted county, a population-based study. Pain 2003;103:199-207.

5 De Mos M, de Bruijn AGJ, Huygen FJ, Dieleman JP, Stricker BH, Sturkenboom MC. The incidence of complex regional pain syndrome: a population-based study. Pain 2007;129:12-20.

6 Moseley GL, Herbert RD, Parsons T, Lucas S, Van Hilten JJ, Marinus J. Intense pain soon after wrist fracture strongly predicts who will develop complex regional pain syndrome: prospective cohort study. J Pain 2014;15:16-23.

7 Beerthuizen A, Stronks DL, Van't Spijker A, Yaksh A, Hanraets BM, Klein J, et al. Demographic and medical parameters in the development of complex regional pain syndrome type 1 (CRPS1): prospective study on 596 patients with a fracture. Pain 2012;153:1187-92.

8 Goebel A, Barker $\mathrm{CH}$, Turner-Stokes $\mathrm{L}$, et al. Complex regional pain syndrome in adults: UK guidelines for diagnosis, referral and management in primary and secondary care. RCP, 2012. www.rcplondon.ac.uk/resources/complex-regional-pain-syndrome-guidelines.

9 Harden RN, Oaklander AL, Burton AW, Perez RS, Richardson K, Swan M, et al. Complex regional pain syndrome: practical diagnostic and treatment guidelines. 4th edition. Pain Med 2013;14:180-229.

10 Allen G, Galer BS, Schwartz L. Epidemiology of complex regional pain syndrome: a retrospective chart review of 134 patients. Pain 1999;80:539-44.

11 De Mos M, Huygen FJ, van der Hoeven-Borgman M, Dieleman JP, Ch Stricker BH, Sturkenboom MC. Outcome of the complex regional pain syndrome. Clin J Pain 2009;25:590-7. 
Box 2: Examples of how to explain to patients about "relaxation, pacing, and goal setting" for self management of chronic pain

Relaxation

Do you remember when we talked about how patients with chronic pain often experience a vicious circle, whereby pain leads to anxiety and tension, which in turn can make pain worse?

So, how can we break that circle? There are several relaxation techniques that might prove helpful. One particularly simple technique is called "relaxation breathing." When people are in pain and anxious, their breathing pattern often changes, becoming fast and shallow. If you recognise that you are becoming tense and anxious you can try to reduce those feelings by trying to breathe slow and deep while keeping your focus on your breathing. You can also try what you already know that helps you relax, such as listening to music or walking.

\section{Pacing}

Many people with chronic pain find themselves reducing their activities. Pacing is about breaking this pattern by increasing gradually what you can do. The first step is to figure out your "baseline time." Think about one activity you would like to be able to do for longer. Note down how long you can do that activity comfortably. Divide that time by 2 . For instance, if you can walk comfortably for 10 minutes your baseline time will be 5 minutes. So, you will try to walk for 5 minutes a couple of times every day. After a week or so you can try walking 7 or 8 minutes a few times daily and progressively increase your walking distance while remaining comfortable.

Goal setting

Goal setting, just like pacing, is about gradually increasing the activities you are able to do. The goals you choose should be reasonable (that is, not impossible) and important to you and traceable. Goals can be things like getting back in touch with someone who was important to you. They can also be complex and made up of several subtasks. For example, getting back to work might require using the pacing technique we had discussed before to increase your ability to perform some of the tasks required in your job, such as typing or climbing stairs.

12 Harden RN, Bruehl S, Perez RS, Birklein F, Marinus J, Maihofner C, et al. Validation of proposed diagnostic criteria (the "Budapest Criteria") for complex regional pain syndrome. Pain 2010;150:268-74.

13 O'Connell NE, Wand BM, McAuley J, Maston L, Lorimer Moseley G. Interventions for treating pain and disability in adults with complex regional pain syndrome. Cochrane Database Syst Rev 2013;4:CD009416.

14 Dworkin RH, O'Connor AB, Audette J, Baron R, Gourlay GK, Haanpää ML, et al. Recommendations for the pharmacological management of neuropathic pain: an overview and literature update. Mayo Clin Proc 2010;85:S3-S14.
15 Centre for Clinical Practice at NICE (UK). Neuropathic pain: the pharmacological management of neuropathic pain in adults in non-specialist settings. NICE (UK), 2010. www.ncbi.nlm.nih.gov/books/NBK82991/.

Accepted: 07 April 2014

Cite this as: BMJ 2014;348:g3683

(c) BMJ Publishing Group Ltd 2014 


\section{Figure}
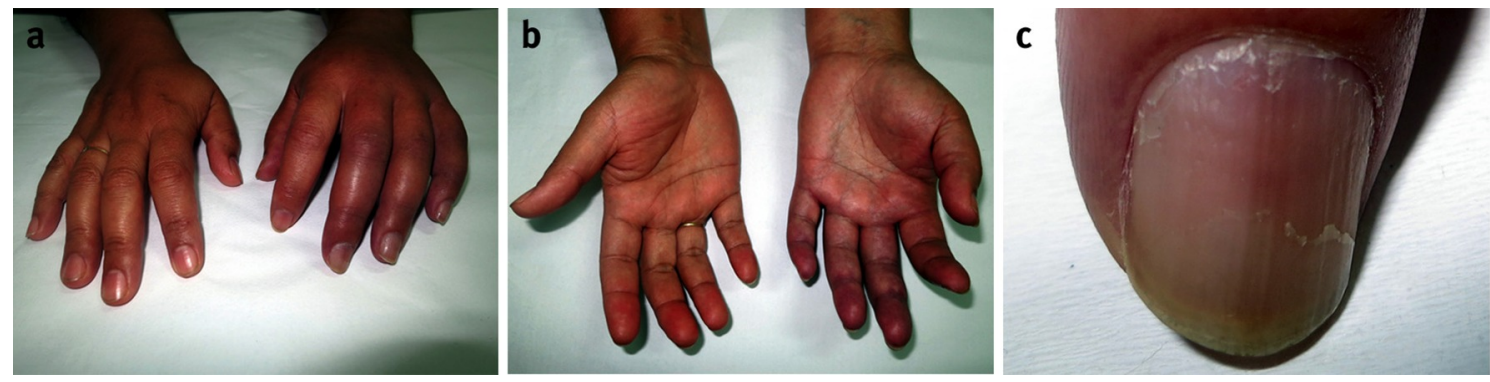

Complex regional pain syndrome of left hand showing oedema with brawny discoloration, shiny skin, and cyanotic fingers ( $a$ and $b$ ); brittle ridged fingernails (c) 